\title{
Cost-Effective Brillouin Optical Time-Domain Analysis Sensor Using a Single Optical Source and Passive Optical Filtering
}

\author{
H. Iribas, J. Urricelqui, J. Mariñelarena, M. Sagues, and A. Loayssa \\ Electrical and Electronic Engineering, Public University of Navarre, 31006 Pamplona, Spain \\ Correspondence should be addressed to A. Loayssa; alayn.loayssa@unavarra.es
}

Received 9 March 2016; Accepted 19 May 2016

Academic Editor: Romeo Bernini

Copyright ( 2016 H. Iribas et al. This is an open access article distributed under the Creative Commons Attribution License, which permits unrestricted use, distribution, and reproduction in any medium, provided the original work is properly cited.

\begin{abstract}
We present a simplified configuration for distributed Brillouin optical time-domain analysis sensors that aims to reduce the cost of the sensor by reducing the number of components required for the generation of the two optical waves involved in the sensing process. The technique is based on obtaining the pump and probe waves by passive optical filtering of the spectral components generated in a single optical source that is driven by a pulsed RF signal. The optical source is a compact laser with integrated electroabsorption modulator and the optical filters are based on fiber Bragg gratings. Proof-of-concept experiments demonstrate $1 \mathrm{~m}$ spatial resolution over a $20 \mathrm{~km}$ sensing fiber with a $0.9 \mathrm{MHz}$ precision in the measurement of the Brillouin frequency shift, a performance similar to that of much more complex setups. Furthermore, we discuss the factors limiting the sensor performance, which are basically related to residual spectral components in the filtering process.
\end{abstract}

\section{Introduction}

Brillouin fiber sensors have been extensively studied over the last two decades. Among them, the Brillouin optical time-domain analysis (BOTDA) technique is probably the most widely used approach, where a pump pulse interacts with a counterpropagating continuous wave $(\mathrm{CW})$ probe in a sensing fiber. BOTDA sensors are able to provide high accuracy in distributed measurements of temperature and strain over large structures. This fact makes them particularly interesting for a large set of industrial sectors, including oil and gas pipe monitoring, assessment of high voltage cables, railway inspection, and many other structural health monitoring applications.

In this context, a significant number of research groups have focused their work on extending and improving the performance of BOTDA sensors, for instance, extending their range to hundreds of kilometers [1], improving their spatial resolution to millimeters [2], or reducing the measurement time to perform dynamic measurements $[3,4]$. However, in many potential applications, the most important limitation to the widespread practical use of BOTDA sensors is their cost rather than their performance. This is because most BOTDA implementations, in order to generate the optical waves involved in the technique, use expensive components such as multiple electrooptic modulators (EOM), lasers, or semiconductor optical amplifiers (SOA). As a consequence, this limits their real-world applications, which are usually bound to industrial sectors where the cost of the sensor is not the main concern, such as in the field of oil and gas.

Thus, in recent years, a line of research in these sensors has opened with the purpose of simplifying the technique in order to achieve cost-effective commercial systems that can compete with other less-costly technologies like, for instance, Raman sensors for temperature monitoring. Examples of contributions intended to reduce the cost of BOTDA sensors include the deployment of offset locking [5] or time-division pump-probe generation using direct modulation of a laser source $[6,7]$, which reduce the number of components removing one SOA or one EOM from the sensor scheme.

In this work, we present an alternative BOTDA sensor setup which is focused on reducing the potential cost of the sensor by obtaining all the optical waves involved in the technique by passive optical filtering of the spectral components generated in a single optical source that is driven by a pulsed RF signal, thereby obtaining a further reduction of used components and avoiding the need for any EOM or 


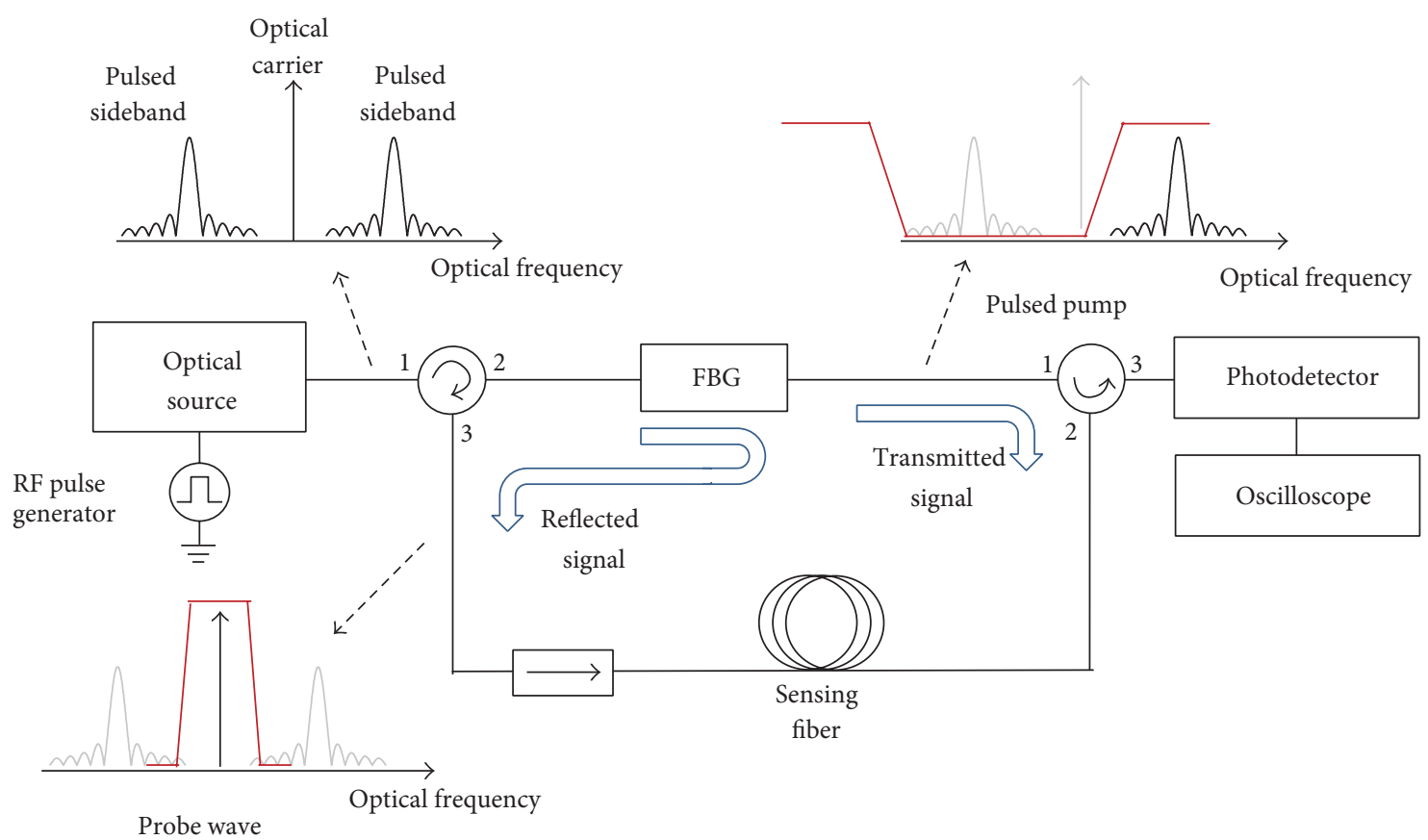

FIGURE 1: Schematic representation of the fundamentals of the technique. The spectra of the signals involved in the setup (in black) and the filter response (red) are sketched in the figure. The pulsed nature of the sidebands of the modulation is graphically represented by a sync spectrum.

SOA in the setup. Note that this paper is an extended version of work presented in [8].

\section{Fundamentals}

The basic principle of the proposed sensor setup is schematically depicted in Figure 1 . The first step is to drive an optical source with a pulse RF signal in order to generate an optical signal comprising a carrier and two pulsedmodulated sidebands. Both the $\mathrm{CW}$ probe wave and the pulsed pump wave required for the BOTDA are derived from this modulated optical signal: the optical carrier of the modulation will act as the CW probe wave, whereas one of the pulsed sidebands of the modulated signal will act as the pump wave. Simple passive filtering is used to separate both waves, as it is described below.

The modulated output of optical source is directed using a circulator to the upper branch of the setup. Here, the signal encounters an optical filter, tuned in such way that one of the two pulsed sidebands of the modulation goes through it, whereas the optical carrier is reflected backwards, returning to the circulator, where it is redirected into the fiber under test. Simultaneously, the pulsed sideband in the upper branch is injected from the other end of the fiber using a second circulator. In this way, both signals counterpropagate in the fiber, where Brillouin interaction takes place. The frequency of the microwave oscillator used to generate the pulsed RF signal is selected to be near the Brillouin frequency shift (BFS) of the fiber, so that the pump pulses can induce Brillouin amplification on the optical carrier. Then, the resultant probe wave is directed to a baseband photoreceiver and the detected electrical signal is captured in a digital oscilloscope. Finally, the measurement is repeated as the modulation frequency is swept, so that the Brillouin gain spectrum (BGS) of the fiber can be reconstructed.

For the optical source, the best option is to deploy a distributed feedback laser (DFB) monolithically integrated with electroabsorption modulator (EAM). The use of such externally modulated laser (EML) compact device, typically in the form of a transmitter optical subassembly (TOSA), has become a standard in optical communications. There is Multisource Agreement (MSA) within the industry that has dramatically brought down the cost of these devices in the market. Therefore, we can use in our technique an EML TOSA designed for $10 \mathrm{~Gb} / \mathrm{s}$ operation and generate all the necessary optical signal at a cost comparable to that of a single DFB laser source.

\section{Experimental Setup}

In order to study the capabilities of the proposed technique, the experimental setup depicted in Figure 2 was assembled. The optical source was a $1551.7 \mathrm{~nm}$ DFB laser source (Emcore 1754C C-band DWDM laser) followed by an integrated EAM (JAE FOEA-310-001). This is driven by high extinction ratio (ER) RF pulses obtained using a microwave oscillator followed by a microwave switch (Miteq SW1-040120RI1NF), which is driven by a pulse generator. In this way, the RF pulse shape is directly translated to the optical domain by the modulator [9]. Low cost commercial microwave switches are available with extremely high isolation and very fast responses. As a result, in our setup it is possible to obtain inexpensive and fast optical pulses (below $1 \mathrm{~ns}$ rise time) with an extremely high ER (>60 dB) 


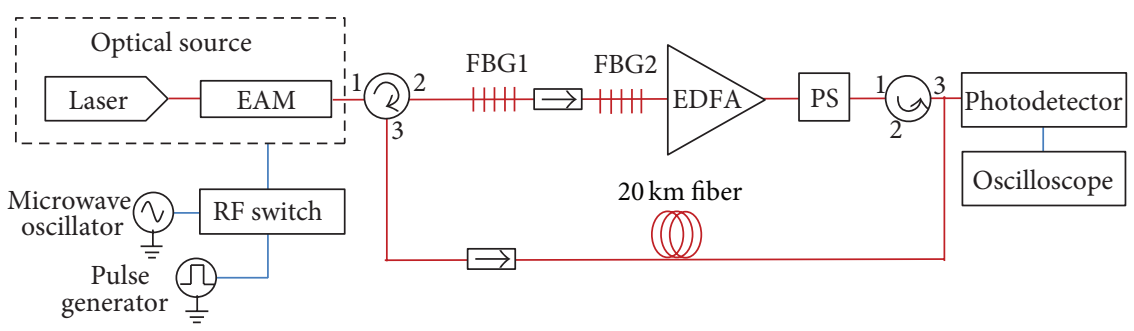

FIGURE 2: Experimental setup for the low cost BOTDA sensor based on pulsed ODSB modulation and passive optical filtering.

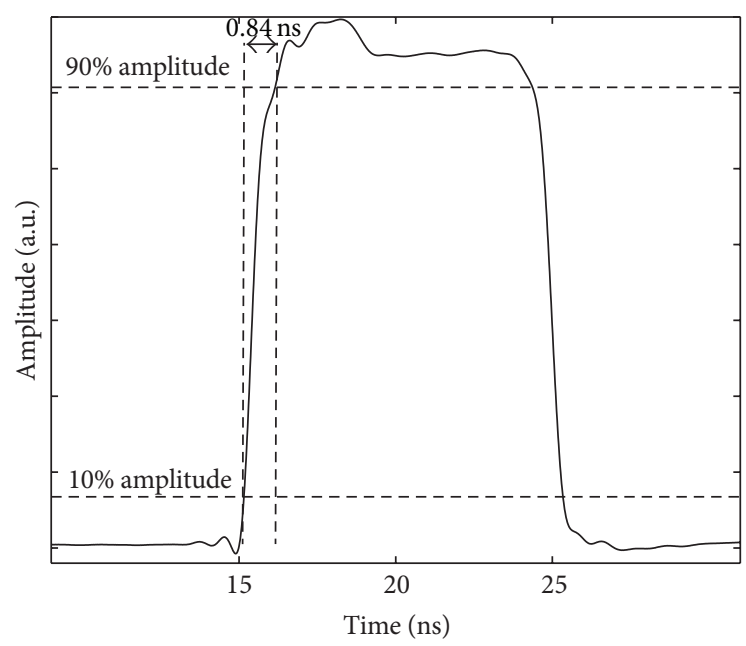

FIGURE 3: Obtained optical pump pulse at the beginning of the fiber at $f=10830 \mathrm{GHz}$.

[9]. Figure 3 shows the temporal shape of the obtained pulse.

It should be noted that due to the small power differences in frequency response of the microwave generator and the microwave switch, pulse amplitude changes slightly at different frequencies. However, this can be easily characterized and compensated in the measurements results.

Once the modulated optical signal is obtained at the output of the optical source, it is directed using a circulator to the upper branch of the setup, where it is filtered employing two narrowband fiber Bragg gratings (FBG). Figure 4(a) shows the optical spectrum obtained at the output of the optical EML source and the transfer function of the both filters. The first FBG was tuned so that the CW optical carrier is reflected, while the pulsed sidebands of the modulation are transmitted. The second FBG was tuned so that the pulsed upper sideband of the modulation is transmitted, while the other sideband and the remains of the CW optical carrier are reflected, so that a gain-based BOTDA sensor was arranged. However, the technique can also be applied to lossbased BOTDA sensors, by simply selecting the lower pulsed sideband of the modulation.

The pulsed pump wave at the output of the second FBG is amplified with an erbium-doped fiber amplifier (EDFA) to a peak power of $20 \mathrm{dBm}$, a level close to the limit before modulation instability (MI) becomes significant [10]. Before being launched into the sensing fiber, the polarization of the pulsed pump is randomized with a polarization scrambler, so as to reduce polarization-mismatching-induced fluctuations on the signal. Note that instead of using a polarization scrambler, a polarization switch or a passive polarization scrambler [11] could be deployed to further reduce the final cost of the sensor setup. Finally, after interaction with the pump pulse along the fiber, the probe signal is detected in a $125-\mathrm{MHz}$ photoreceiver and captured in a digitizer.

Notice that the optical filtering deployed in the setup is not ideal; hence there is always going to be some degree of crosstalk between the generated optical signals in both branches of the setup. This is highlighted in Figure 4(b), where the optical spectrum of the pump pulse in the upper branch of the setup after being filtered and amplified is shown. Note that it is possible to see residuals of the optical carrier and also other pulsed sidebands alongside the desired optical component. Moreover, Figure 4(c) shows the optical spectrum of the probe wave at the lower branch of the setup at the beginning of the test fiber, where not solely appears the probe wave, but also residuals of pulsed spectral components. As we will discuss later, these residual signals impair the performance of the sensor.

\section{Experimental Results and Discussion}

In order to analyze the performance of the system, distributed temperature measurements were performed over a $20 \mathrm{~km}$ length of standard single-mode (ITU G.652) fiber. The pulse duration was set to $10 \mathrm{~ns}$, corresponding to approximately $1 \mathrm{~m}$ resolution. The final $30 \mathrm{~m}$ of the fiber was placed loose in a climatic chamber, while the rest was held at room temperature in a spool. The modulation frequency was swept at $2 \mathrm{MHz}$ steps and 4096 averages were used to obtain the final traces.

The temperature of the climatic chamber (hot spot) was modified with the purpose of evaluating the system performance. The spectra measured at the heated section of the fiber are depicted in Figure 5(a). As it was expected, the measured spectra are shifted in frequency as the temperature is raised. The BFS along the fiber was calculated by performing a mathematical fit to a Voigt profile. The relation between temperature and BFS in the fiber is depicted in Figure 5(b), where a linear regression is performed to obtain a temperature coefficient of $1.0756 \mathrm{MHz} /{ }^{\circ} \mathrm{C}$.

Also, we analyzed the performance of the sensor in terms of spatial resolution and measurement precision. Figure 6(a) 


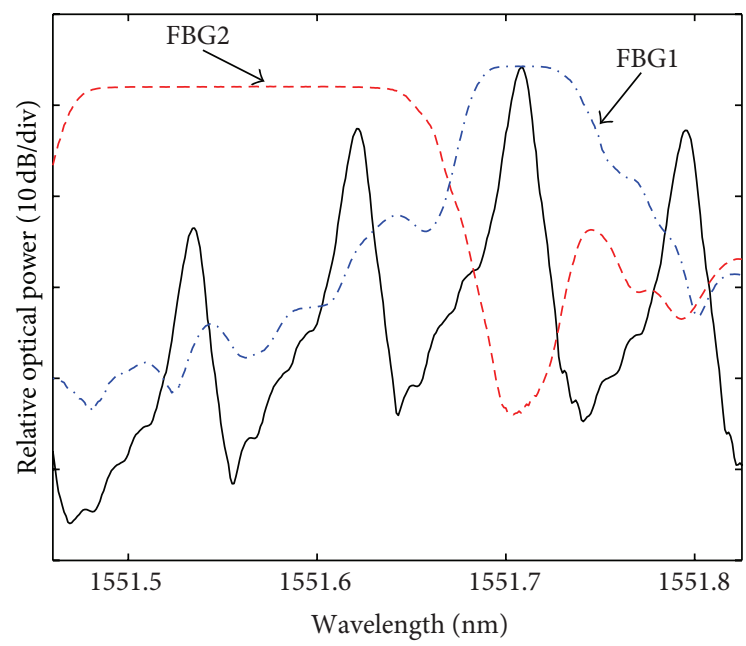

(a)

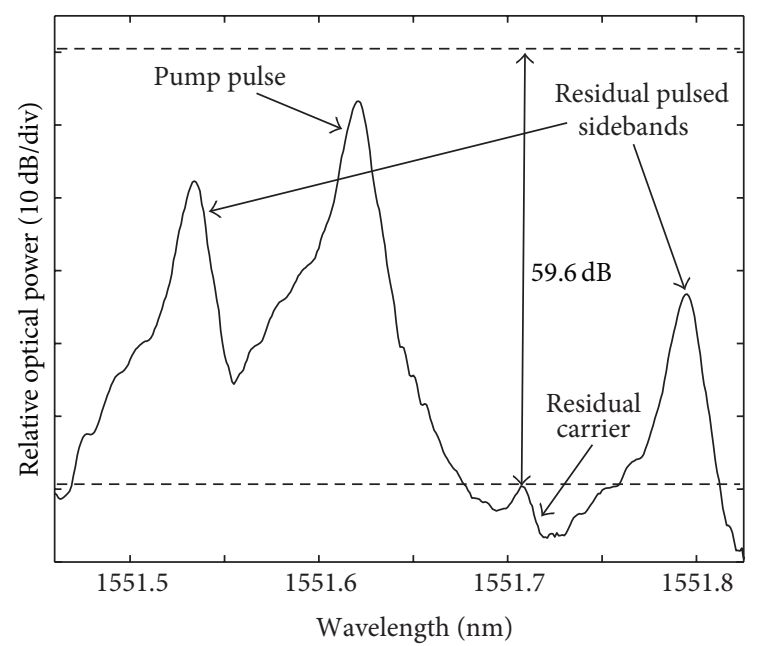

(b)

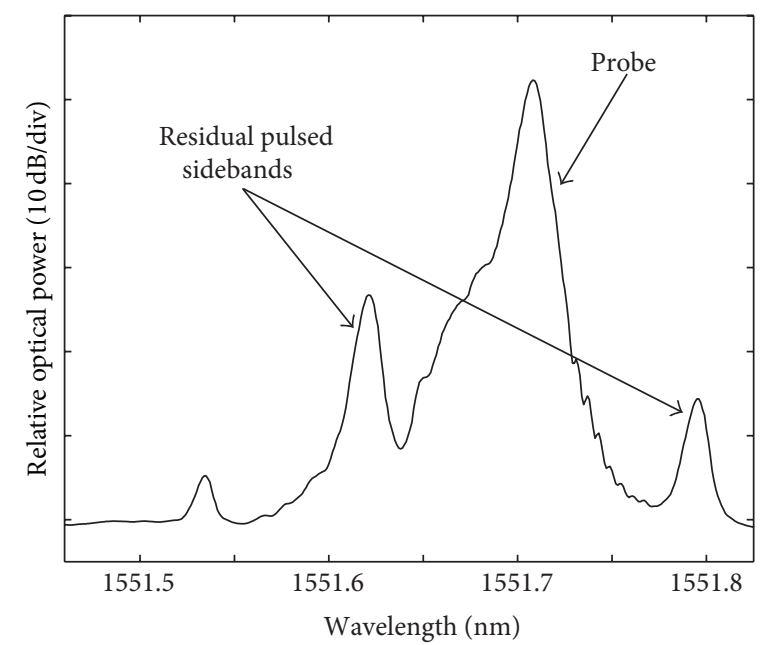

(c)

FIGURE 4: Optical spectrum of the optical signal at $f=10830 \mathrm{GHz}$ in different positions of the setup (black solid) (a) at the output of the DFB-EAM module, transfer function of the FBG1 (blue dash-dot), and transfer function of the FBG2 (red dashed), (b) at the input of the EDFA in the upper branch of the setup, and (c) at the beginning of the fiber in the lower branch of the setup.

depicts the evolution of the measured BFS at the end of the fiber for different temperatures. The $30 \mathrm{~m}$ section corresponding to the hot spot is clearly visible and a fast transition between the heated section and the rest of the fiber is also observed. The spatial resolution was confirmed to be $1 \mathrm{~m}$ by measuring the rise time between two adjacent sections of the fiber at different temperatures. This is shown in Figure 6(b), where the rise time equivalent length, in meters, for the transition between the heated and the room temperature sections of the fiber is calculated.

The measurement precision was obtained by performing series of consecutive measurements for stable temperature conditions. From these measurements, the 2-sigma BFS measurement precision was found to be $0.9 \mathrm{MHz}$. Furthermore, based on the obtained results, the figure of merit (FoM) of this BOTDA setup was calculated [12], obtaining a value of 1.1. This FoM turns out to be of the same order of more complex BOTDA setups that deploy several EOMs or SOAs to generate the required optical signals [12].
We also studied the factors that were limiting our BOTDA setup performance, particularly those associated with the crosstalk between the optical signals in both sensor branches that we discussed before. Figure 7 highlights the deleterious effect of the pulsed sidebands residuals present in the probe wave (Figure 4(c)) on the measured BOTDA trace. Notice that there is an abrupt loss of signal at the middle of the fiber. This effect was found to be originated by the residuals of the pulse traveling along the fiber in the direction of the probe wave.

The arrival of this pulse at the output of the fiber saturates the photoreceiver amplifier invalidating the measurement until it returns to a stabilized situation after the pulse passes. The inset of Figure 7 expands the signal-loss area, where it can be seen that for a time duration equivalent to approximately $60 \mathrm{~m}$ the measurement is corrupted.

However, this limitation is not really serious because it can be overcome in a number of ways, depending on the measurement configuration that is being deployed. 


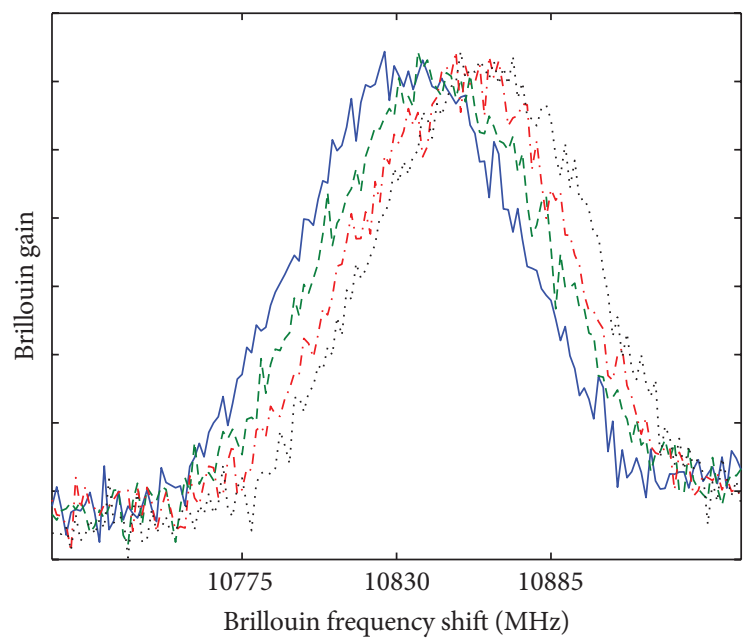

(a)

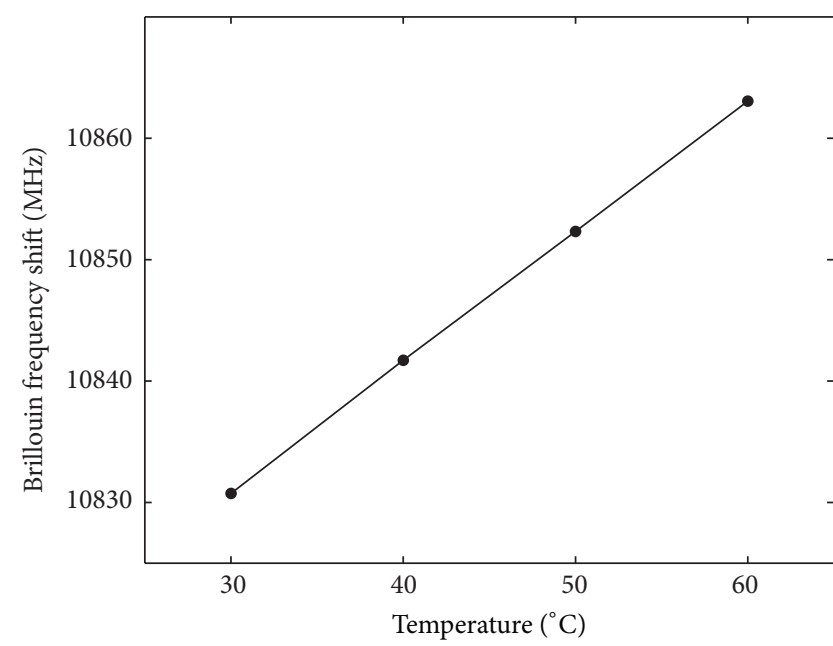

(b)

FIGURE 5: (a) Brillouin gain spectra measured at the heated location of the fiber, as the temperature is raised in $10^{\circ} \mathrm{C}$ steps. (b) Calculated $\mathrm{BFS}$ as a function of temperature in the climatic chamber (symbols) and linear regression (solid line).

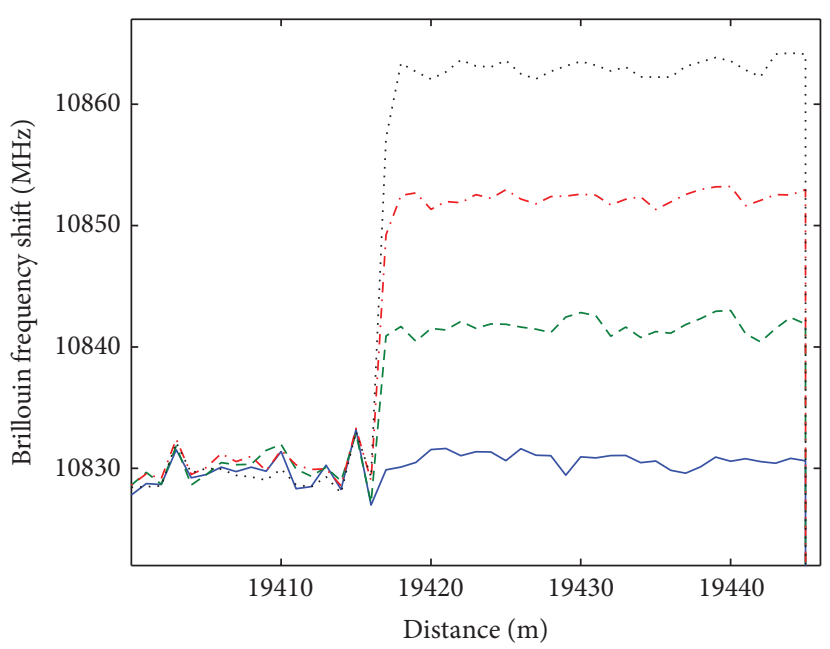

(a)

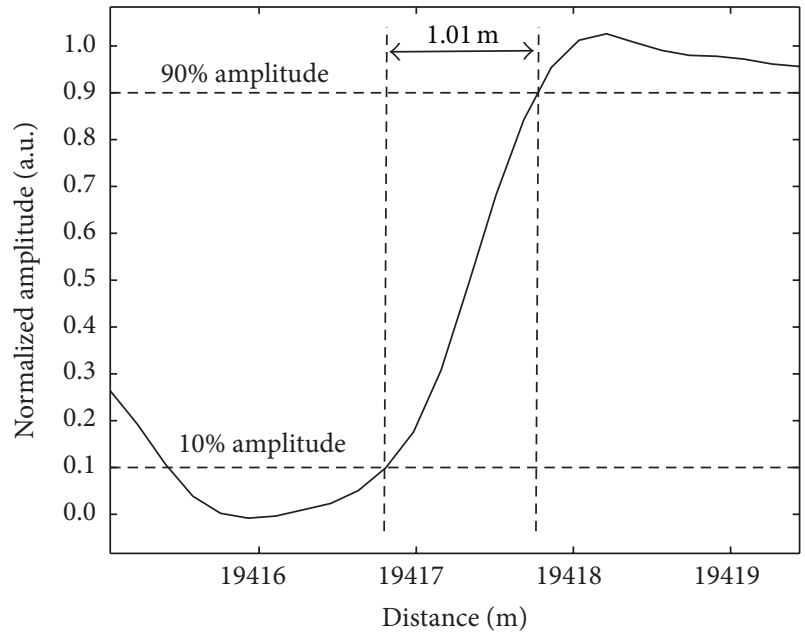

(b)

FIGURE 6: (a) Calculated BFS as a function of distance at the final locations of the fiber, as the temperature is raised in the climatic chamber in $10^{\circ} \mathrm{C}$ steps. (b) Normalized amplitude of the detected signal at the transition between the heated and the room temperature sections of the fiber.

An obvious solution would be to enhance the optical filters to increase the unwanted components suppression in the probe signal. However, there are practical limits for this approach. On the other hand, BOTDA sensors typically use loop configurations, for example, when measuring a pipeline, in which there is a leading fiber, used to bring the probe wave to the middle of the loop where the sensing fiber starts. Therefore, the problem is solved altogether because the corrupted measurement span would be outside the sensing fiber. Nevertheless, if we are interested in measuring along the full fiber length, we could take into account this fact when the fiber is installed in the structure that is to be monitored by simply installing an extra length of fiber ( 60 meters) in the middle section.
The other limitation that we studied was due to the residuals of the optical carrier on the pump pulse (Figure 4(b)). This residual carrier propagates along the fiber with the pump pulse, generating a Rayleigh backscattered (RB) signal that reaches the receiver together with the probe wave, which has identical wavelength, thus leading to interference and noise. This noise can be quantified by considering the total optical field that reaches the photoreceiver, which can be approximated by

$$
E(t)=E_{\mathrm{Pr}} e^{\left(2 \pi j v_{\mathrm{Pr}} t+\phi_{\mathrm{Pr}}\right)}+E_{\mathrm{RB}} e^{\left(2 \pi j v_{\mathrm{RB}} t+\phi_{\mathrm{RB}}\right)},
$$

where $E_{\mathrm{Pr}}$ and $E_{\mathrm{RB}}$ are the amplitude of the probe wave and $\mathrm{RB}$ components, respectively, $\nu_{\mathrm{Pr}}$ and $\nu_{\mathrm{RB}}$ are the optical 


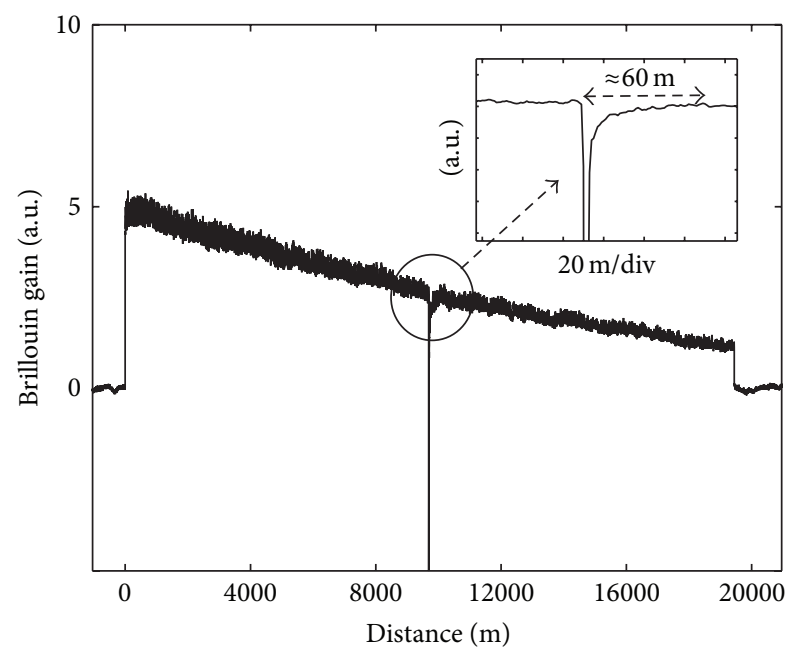

Figure 7: The BOTDA trace at $f=10830 \mathrm{GHz}$ for the $20 \mathrm{~km}$ fiber with the corrupted middle section of the fiber in a zoomed area.

frequency of probe and $\mathrm{RB}$ waves, and $\phi_{\mathrm{Pr}}$ and $\phi_{\mathrm{RB}}$ are the phases of the probe wave and the RB.

The total intensity (optical power) detected at the photoreceiver includes a noise term generated by the RB beating with the probe wave, which can be written as

$$
\left.I(t)\right|_{\mathrm{RB}-\mathrm{Pr}}=2 E_{\mathrm{Pr}} E_{\mathrm{RB}} \cos (2 \pi \Delta \nu t+\Delta \Phi),
$$

where $\Delta v=\Delta \nu_{\mathrm{RB}}-\Delta \nu_{\mathrm{Pr}}$ is the frequency detuning between probe and RB waves and $\Delta \Phi$ is the phase difference between probe and $\mathrm{RB}$, which can be assumed to be a random variable with uniform distribution between $-\pi$ and $\pi$. This is a stationary random process, whose autocorrelation function is

$$
R(\tau)=2 P_{\mathrm{Pr}} P_{\mathrm{RB}} \cos (2 \pi \Delta v \tau),
$$

where $P_{\mathrm{Pr}}$ and $P_{\mathrm{RB}}$ are the probe power and RB power at the receiver, respectively. As (2) is a random process with zero mean, the standard deviation of the RB-probe beating noise can be calculated as

$$
\sigma_{\mathrm{RB}-\mathrm{Pr}}=\sqrt{R(0)}=\sqrt{2 P_{\mathrm{Pr}} P_{\mathrm{RB}}} .
$$

Therefore, the total noise power at the photoreceiver output can be expressed as follows:

$$
\sigma_{T}^{2}=\sigma_{\mathrm{th}}^{2}+\sigma_{\mathrm{sh}}^{2}+\sigma_{\mathrm{RB}}^{2}
$$

where $\sigma_{\mathrm{th}}^{2}$ and $\sigma_{\mathrm{sh}}^{2}$ are variance of the thermal and shot noise at the photoreceiver, respectively, and $\sigma_{\mathrm{RB}}^{2}$ is the noise variance calculated using (4), induced by the Rayleigh backscattering beating with the probe wave. Other noises have been neglected in this expression because they tend to be much less significant.

In order to estimate the impact of the RB-probe beating noise in the system, the optical powers of the probe wave and $\mathrm{RB}$ arriving at the photoreceiver were measured, giving values of $-14 \mathrm{dBm}$ and $-70 \mathrm{dBm}$, respectively. Applying (4) to these experimentally obtained results and using the value of the responsivity of the photoreceiver, we obtained a value of $3.6 \mathrm{mV}$ for the standard deviation of the RB-probe beating noise.

This value was double-checked using (5). First the total noise was measured with the EDFA in the upper branch turned off, giving a standard deviation of noise (thermal and shot) of $2.6 \mathrm{mV}$. Then the EDFA was turned on and the total standard deviation of noise was measured to be $4.9 \mathrm{mV}$. Using (5), this translates to added noise with standard deviation of $4.1 \mathrm{mV}$. This agrees well with the value calculated using the model in (1)-(4). Therefore, it is confirmed that the main system noise is originated by the residual of the optical carrier in the pump pulse wave, which introduces a $5.5 \mathrm{~dB}$ penalty in signal-to-noise ratio (SNR).

The noise added by RB is directly proportional to the optical carrier suppression, as it is highlighted by (4). Therefore, in order to increase the detected SNR, one option would be to apply more filtering, for instance, adding other FBG in the upper branch of the setup to enhance the optical carrier suppression.

For example, adding $10 \mathrm{~dB}$ additional suppression to the carrier, which is relatively simple to achieve, would reduce the SNR penalty to less than a decibel.

\section{Conclusion}

A simplified configuration for Brillouin distributed sensors has been proposed and demonstrated. The sensor relies on passive optical filtering of the spectral components generated in a single RF-pulsed optical source. This reduces the number of the components required to implement the sensor making it a cost-effective alternative to conventional BOTDA setups. The capabilities of the technique have been analyzed performing distributed temperature measurements over a $20 \mathrm{~km}$ length of fiber with high precision and resolution, demonstrating a performance comparable to more complex setups. Furthermore, the factors limiting this performance have been studied and the way to overcome them has been outlined. Future work will focus on improving the performance of the sensor and further simplifying its implementation, so that BOTDA sensors can reach applications and industries from which they are now excluded because of cost concerns.

\section{Competing Interests}

The authors declare that they have no competing interests.

\section{Acknowledgments}

The authors wish to acknowledge the financial support from the Spanish Ministerio de Ciencia e Innovación through the Project TEC2013-47264-C2-2-R, FEDER funds, and Universidad Pública de Navarra.

\section{References}

[1] S. Le Floch, F. Sauser, M. Llera, and E. Rochat, "Novel brillouin optical time-domain analyzer for extreme sensing range using 
high-power flat frequency-coded pump pulses," Journal of Lightwave Technology, vol. 33, no. 12, pp. 2623-2627, 2015.

[2] R. Cohen, Y. London, Y. Antman, and A. Zadok, "Brillouin optical correlation domain analysis with 4 millimeter resolution based on amplified spontaneous emission," Optics Express, vol. 22, no. 10, pp. 12070-12078, 2014.

[3] R. Bernini, A. Minardo, and L. Zeni, "Dynamic strain measurement in optical fibers by stimulated brillouin scattering," Optics Letters, vol. 34, no. 17, pp. 2613-2615, 2009.

[4] J. Urricelqui, A. Zornoza, M. Sagues, and A. Loayssa, "Dynamic BOTDA measurements based on brillouin phase-shift and RF demodulation," Optics Express, vol. 20, no. 24, pp. 26942-26949, 2012.

[5] Y. Li, X. Bao, F. Ravet, and E. Ponomarev, "Distributed Brillouin sensor system based on offset locking of two distributed feedback lasers," Applied Optics, vol. 47, no. 2, pp. 99-102, 2008.

[6] K. Y. Song and S. Yang, "Simplified Brillouin optical time-domain sensor based on direct modulation of a laser diode," Optics Express, vol. 18, no. 23, pp. 24012-24018, 2010.

[7] J. G. Hong and K. Y. Song, "Simplified brillouin optical timedomain analysis with extended measurement range by frequency dithering," in Proceedings of the 23rd International Conference on Optical Fibre Sensors, vol. 9157 of Proceedings of SPIE, Santander, Spain, June 2014.

[8] H. Iribas, J. Urricelqui, J. Marinelarena, M. Sagues, and A. Loayssa, "Simplified Brillouin sensor for structural health monitoring applications based on passive optical filtering," in 24th International Conference on Optical Fibre Sensors, vol. 9634 of Proceedings of SPIE, The International Society for Optical Engineering, 2015.

[9] A. Zornoza, D. Olier, M. Sagues, and A. Loayssa, "Brillouin distributed sensor using RF shaping of pump pulses," Measurement Science and Technology, vol. 21, no. 9, Article ID 094021, 2010.

[10] M. N. Alahbabi, Y. T. Cho, T. P. Newson, P. C. Wait, and A. H. Hartog, "Influence of modulation instability on distributed optical fiber sensors based on spontaneous brillouin scattering," Journal of the Optical Society of America B: Optical Physics, vol. 21, no. 6, pp. 1156-1160, 2004.

[11] S. Diaz, S. F. Mafang, M. Lopez-Amo, and L. Thévenaz, "A highperformance optical time-domain brillouin distributed fiber sensor," IEEE Sensors Journal, vol. 8, no. 7, pp. 1268-1272, 2008.

[12] M. A. Soto and L. Thévenaz, "Modeling and evaluating the performance of brillouin distributed optical fiber sensors," Optics Express, vol. 21, no. 25, pp. 31347-31366, 2013. 


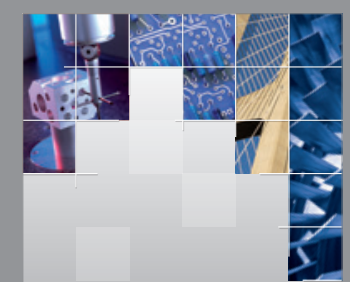

\section{Enfincering}
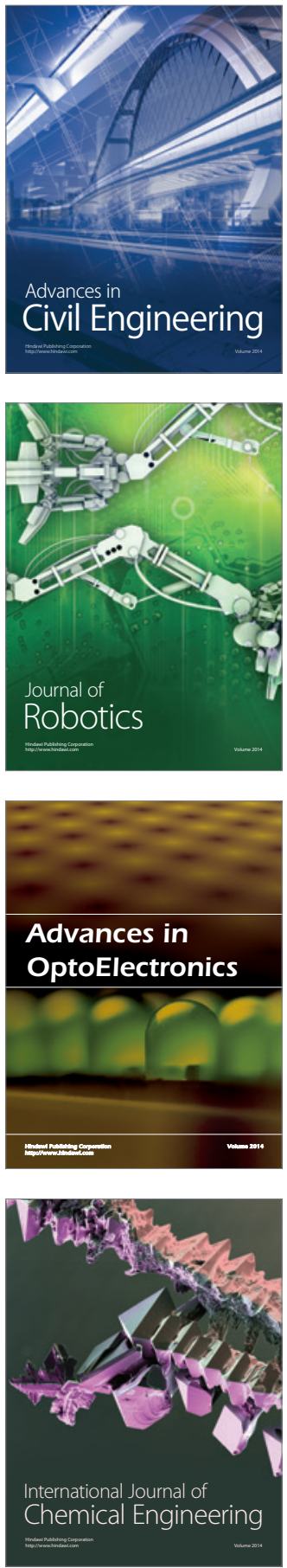

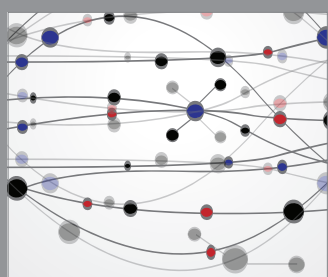

The Scientific World Journal

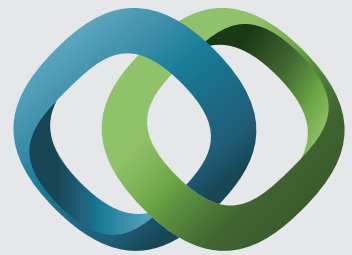

\section{Hindawi}

Submit your manuscripts at

http://www.hindawi.com
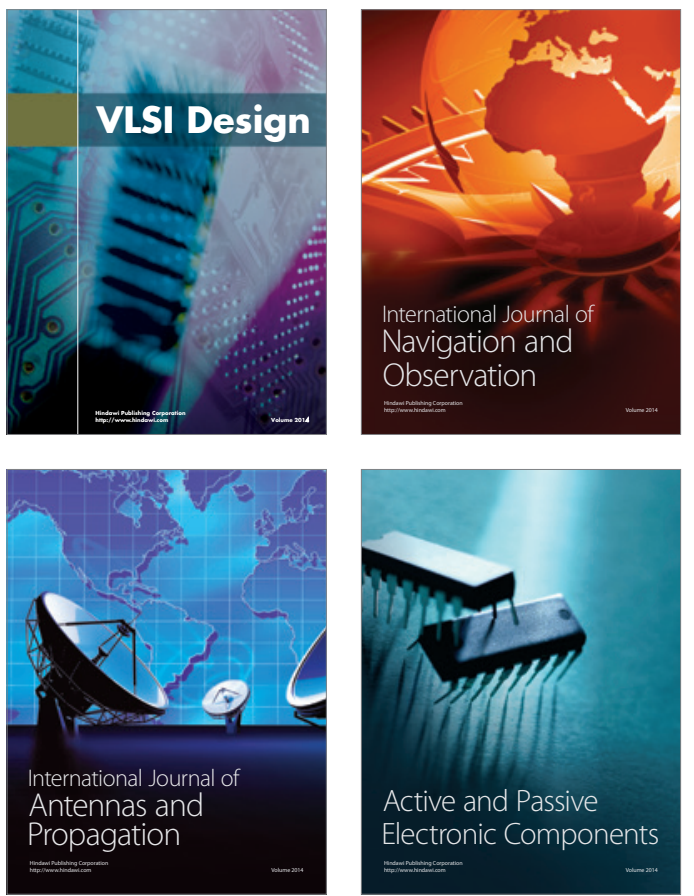
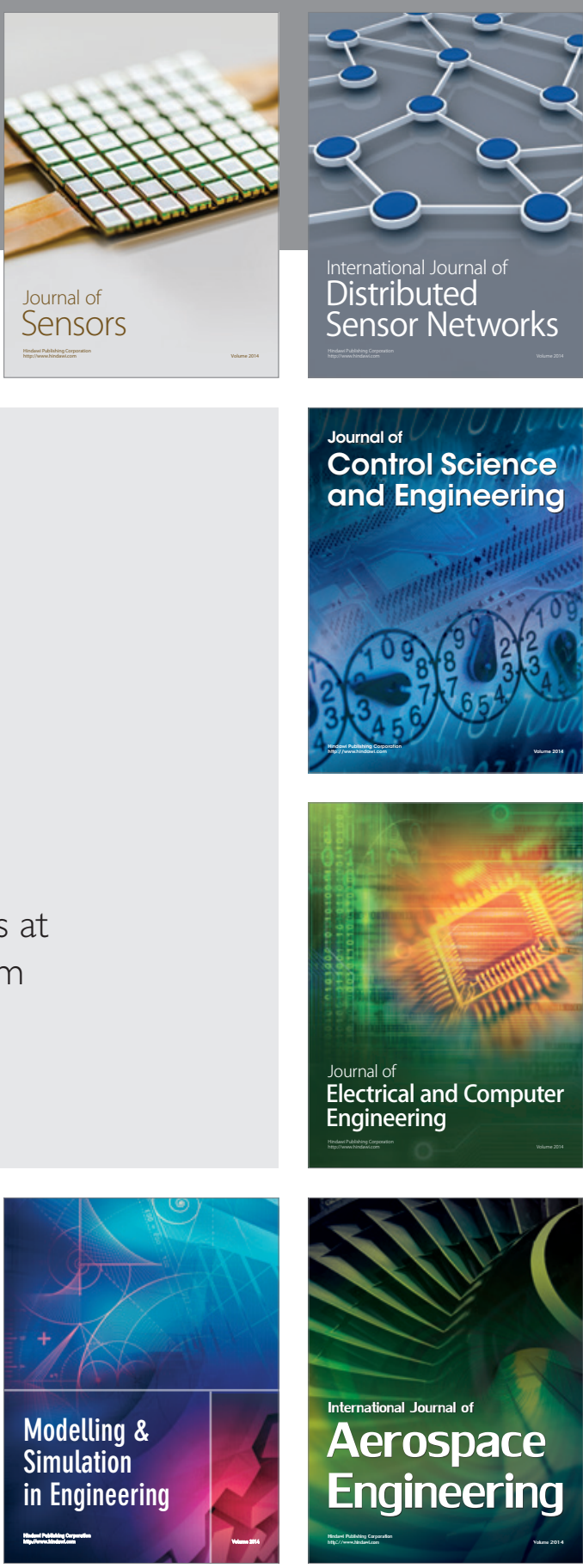

International Journal of

Distributed

Sensor Networks

Journal of

Control Science

and Engineering
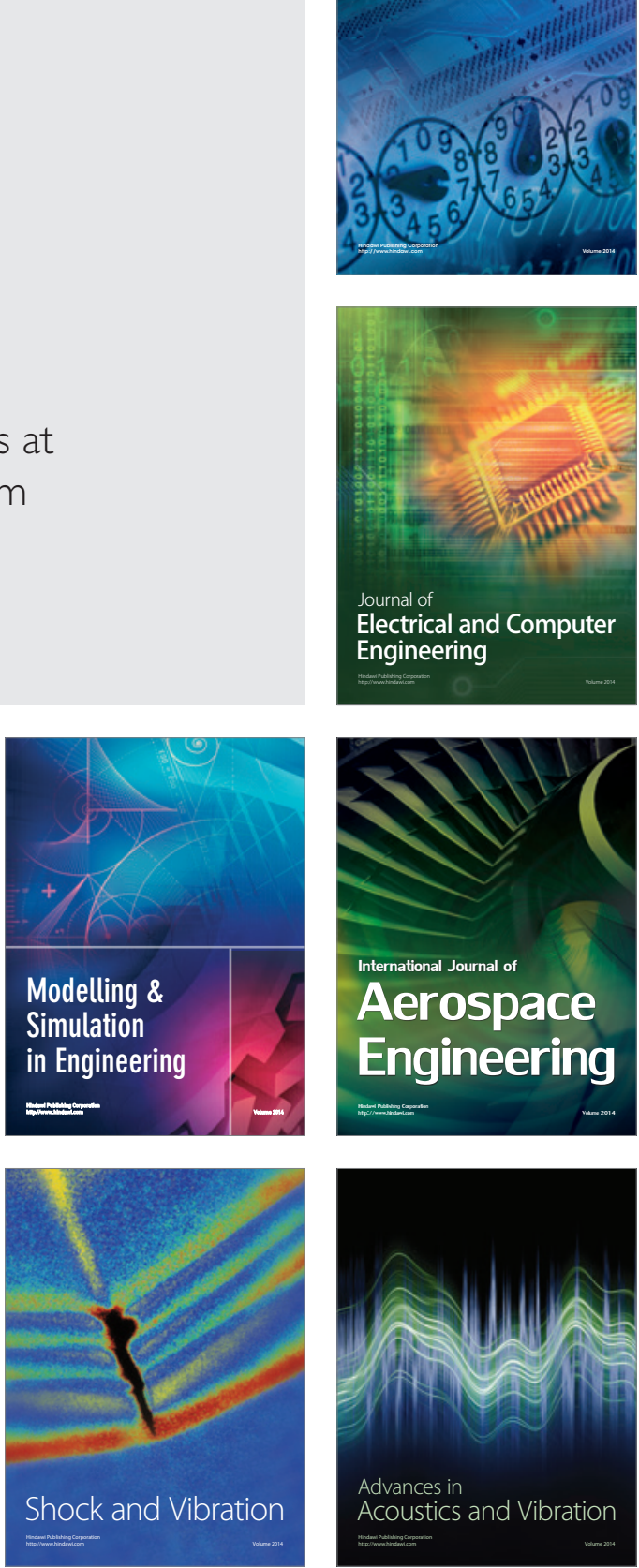